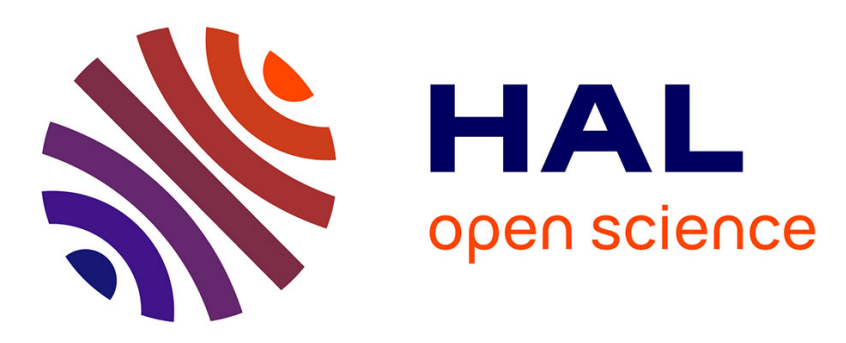

\title{
Normalized equilibrium in Tullock rent seeking game
} Eitan Altman, Mandar Datar, Gérard Burnside, Corinne Touati

\section{To cite this version:}

Eitan Altman, Mandar Datar, Gérard Burnside, Corinne Touati. Normalized equilibrium in Tullock rent seeking game. GameNets 2019 - Game Theory for Networks, Apr 2019, Paris, France. pp.109-116, 10.1007/978-3-030-16989-3_8. hal-02291549

\section{HAL Id: hal-02291549 \\ https://hal.inria.fr/hal-02291549}

Submitted on 19 Sep 2019

HAL is a multi-disciplinary open access archive for the deposit and dissemination of scientific research documents, whether they are published or not. The documents may come from teaching and research institutions in France or abroad, or from public or private research centers.
L'archive ouverte pluridisciplinaire HAL, est destinée au dépôt et à la diffusion de documents scientifiques de niveau recherche, publiés ou non, émanant des établissements d'enseignement et de recherche français ou étrangers, des laboratoires publics ou privés. 


\section{Normalized equilibrium in Tullock rent seeking game}

\author{
Eitan Altman, Mandar \\ Matar \\ joint Lab of INRIA and Nokia \\ Bell-Labs \\ LIA, Univ of Avignon, and \\ LINCS, Paris, France \\ Eitan.Altman@inria.fr
}

\author{
Gerard Burnside \\ joint Lab of INRIA and Nokia \\ Bell-Lab, France \\ gerard.burnside@nokia- \\ bell-labs.com
}

\author{
Corinne Touati \\ joint Lab of INRIA and Nokia \\ Bell-Labs, France \\ corinne.touati@inria.fr
}

\begin{abstract}
Games with Common Coupled Constraints represent many real life situations. In these games, if one player fails to satisfy its constraints common to other players, then the other players are also penalised. Therefore these games can be viewed as being cooperative in goals related to meeting the common constraints, and non cooperative in terms of the utilities. We study in this paper the Tullock rent seeking game with additional common coupled constraints. We have succeded in showing that the utilities satisfy the property of diagonal strict concavity (DSC), which can be viewed as an extention of concavity to a game setting. It not only guarantees the uniqueness of the Nash equilibrium but also of the normalized equilibrium.
\end{abstract}

\section{INTRODUCTION}

Games with constraints have long been used for modeling and studying non-cooperative behavior in various areas. This includes road traffic [7, 12] and telecommunications [9]. Various types of constraints may appear in every day game situations; the simplest consisting of orthogonal constraints, where the strategies of the players are restricted independently of each other [15]. A second type of constraints are called Common Coupled Constraints (CCC) [3, $14,15]$ in which all players have a common convex nonorthogonal multi-strategy space. This model can be viewed as constraints that are common to all users. A unilateral deviation of a player from some feasible multi-strategy (one that satisfies the constraints) to another strategy that is feasible for that player, does not result, therefore, in the violation of constraints of other users. CCC have often been used in telecommunications networking problems as well as in power transfer over a smart grid, where capacity constraints of links are naturally common. Games with this type of constraints are a special case of General Constrained Games (GCG) [6], see also [3, 4, 5, 8, 10, 16].

In this paper we study the well known Tullock rent seeking game with Common Coupled Constraints. This game describes contest over resources. Each player bids an amount that she is ready to pay. She then pays an amount proportional to her bid and receives in turn a payoff that is proportional to her bid divided by the sum of bids of all players.

Gamenets 2019 Paris, France

Copyright is held by author/owner(s).
The presence of a capacity constraint results in infinitely many equilibria and we are faced with a question of equilibrium selection. Using Kuhn Tucker conditions to the best response, we can solve a relaxed game instead of the original constrained game, which has however the same equilibria as the original game. The Lagrange multipliers can be interpreted as a shadow cost that a manager sets in order to guarantee that the equilibrium achieved satisfies the constraints. This approach may however be completey unscalable since KKT Theorem does not guarantee that the price per resource unit is the same for all players. In fact, since the Lagrange multipliers are obtained for the best response function, they could depend not only on the player but also on the policy of all other players, rending the approach even less scalable. We are interested in finding such shadow cost which is fixed per resource unit. Such an equilibrium along with a fixed shadow price is called a normalized equilibrium.

The Tullock rent-seeking game has been used recently to model and study several game phenomena in networking. It was used to model contests over timelines in social netorks for maximizing visibility [17]. Each player $i$ controls the rate $\lambda_{i} a_{i}$ of a Poisson process of posts that player $i$ sends into a common timeline of length $K$. This rate is given by a basic popularity rate $\lambda_{i}$ times the acceleration effort (e.g. through advertisement) given by $a_{i}$. Using basic queueing theory, the authors show that the stationary expected number of posts in the timeline originating from player $i$ is given by

$$
K \frac{\lambda_{i} a_{i}}{\sum_{j=1}^{N} \lambda_{j} a_{j}}
$$

This visibility measure is the payoff in Tullock's model, while the cost for acceleration at a rate $a_{i}$ is proportional to $a_{i}$ as in Tullick's model.

Another application of the Tullock rent seeking game is in the study of contests between miners in blockchain [2].

A few words on rent seeking. According to Wikipedia, "In public choice theory and in economics, rent-seeking involves seeking to increase one's share of existing wealth without creating new wealth. Rent-seeking results in reduced economic efficiency through poor allocation of resources, reduced actual wealth-creation, lost government revenue, increased income inequality, and (potentially) national decline."

Wikipedia further describes the origin of the idea: "The idea of rent-seeking was developed by Gordon Tullock in 1967, while the expression rent-seeking itself was coined in 1974 by Anne Krueger[11]. The word "rent" does not refer specifically to payment on a lease but rather to Adam 
Smith's division of incomes into profit, wage, and rent. The origin of the term refers to gaining control of land or other natural resources."

Our first contribution is to show that the utilities satisfy a property that extends concavity to games, and is called Diagonally Strict Concavity. This is shown to imply the existence and uniqueness of a normalized equilibrium. We shall then show that this property further extends to the case of contests over several resources.

\section{A SINGLE RESOURCE}

Consider an $\mathrm{N}$ players game. Player $m$ bids a quantity $x^{m}$. We have minimum constraints $x^{m} \geq \epsilon$ for all $m$.

The payoff from this contest to player $m$ is

$$
P^{m}=\frac{x^{m}}{\sum_{j=1}^{M} x^{j}} .
$$

This comes at a cost of $x^{m} \gamma$ to player $m$ where $\gamma$ is a constant. The utility for player $m$ is thus

$$
U^{m}(x)=\frac{x^{m}}{\sum_{j=1}^{M} x^{j}}-x^{m} \gamma .
$$

THEOREM 1. (i) The utility of player $m$ is concave in its action and is continuous in the actions of other players.

(ii) For any strictly positive value of $\gamma$, the above game has a unique Nash equilibrium in pure policies.

Proof. Direct calculation leads to (i). The existence then directly follows from [15]. Uniqueness is established in [1], see also [18]. Other related uniqueness results in the asymmetric case can be foudn in $[17,19]$.

\section{NORMALIZED EQUILIBRIUM}

The games we have seen so far involved orthogonal constraints. By that we mean that the actions that a player can use do not depend on the actions of other players. We next introduce capacity constraint. We require that the following holds for some contant $V$ :

$$
\sum_{j=1}^{M} x^{j} \leq V
$$

Capacity constraints may represent physical bounds on resources, such as bounded power, or resources that are bounded by regulation. For example, legislation may impose bounds on the power used or on the emission of $\mathrm{CO} 2$ by cars. With the additional capacity constraint, the Nash equilibrium is no more unique and there may in fact be an infinite number of equilibria. We call this the game with capacity constraint.

Let $y$ be an equilibrium in the above game and let $y[-m]$ denote the action vectors of all players other than $m$. By KKT Theorem, since for each $m, U^{m}$ is concave in $x^{m}$, there is a Lagrange multiplier $\lambda^{m}\left(y_{[-m]}\right.$ such that $y^{m}$ maximizes the Lagrangian

$$
L^{m}\left(x^{m}\right)=U^{m}\left(x, y_{[-m]}\right)-\lambda^{m}\left(y_{[-m]}\right)\left(V_{k}-\sum_{j=1}^{M} x^{j}\right)
$$

and

$$
\lambda^{m}\left(y_{[-m]}\right)\left(V-\sum_{j=1}^{M} x^{j}\right)=0
$$

(complementarity property). We call the game with the Lagrangians $L^{m}$ replacing the utilities $U^{m}$ the relaxed game.

The Lagrange multipliers can be interpreted as shadow prices: if a price is set on player $m$ such that when other players are at equilibrium, the player pays $x^{m} \lambda^{m}\left(y_{[-m]}\right)$ for its use of the capacity, then $y$ is an equillibrium in the game with capacity constraints. Yet this pricing is not scalable since for the same use of the resources it may vary from user to user and it further depends on the the chosen equilibrium. For billing purposes one would prefer $\lambda^{m}$ not to depend on $y$ nor on $m$, but to be a constant.

Does there exist a constant Lagrange multiplier $\lambda$ independent of strategies of the payers and of the idendity $m$ of the player, along with an associated equilibrium $y$ for the corresponding relaxed game? If the answer is positive then $y$ is called a normalized equilibrium [15].

Our goal is to establish the existence and uniquenesss of the normalized equilibrium.

\section{DIAGONAL STRICT CONCAVITY}

For a vector of real nonnegative numbers $r$, define

$$
\begin{gathered}
\sigma(x, r)=\sum_{m=1}^{N} r_{m} U^{m}(x) \\
g(x, r)=\left[\begin{array}{c}
r_{1} \frac{\partial}{\partial x_{1}} U^{1}\left(x_{1}, x_{-1}\right) \\
r_{2} \frac{\partial}{\partial x_{2}} U^{2}\left(x_{2}, x_{-2}\right) \\
\vdots \\
r_{N} \frac{\partial}{\partial x_{N}} U^{N}\left(x_{N}, x_{-N}\right)
\end{array}\right]
\end{gathered}
$$

$\sigma$ is called diagonally strict concave (DSC) for a given $r$ if for every distinct $x^{0}$ and $x^{1}$,

$$
\left(x^{1}-x^{0}\right)^{\prime} g\left(x^{0}, r\right)+\left(x^{0}-x^{1}\right)^{\prime} g\left(x^{1}, r\right)>0
$$

Let $\mathrm{G}(\mathrm{x}, \mathrm{r})$ be the Jacobian of $\mathrm{g}(\mathrm{x}, \mathrm{r})$ with respect to $\mathrm{x}$ and let $G_{i, j}$ be $i^{\text {th }}$ row and $j^{\text {th }}$ column of $\mathrm{G}(\mathrm{x}, \mathrm{r})$. Then a sufficient condition for $\sigma$ to be diagonally strict concave for some $r$ is that for all $x,\left[G(x, r)+G^{\prime}(x, r)\right]$ is negative definite.

Our interest in diagonally strict concave utility functions is due to the following properties of games possesing such utilities.

TheOREM 2. (Theorem 4 from [15]) Let $\sigma$ be diagonally strict concave for some $r$. Then there exists a unique normalized equilibrium.

\section{PROOF OF DSC}

In this section we establish that the Tullock game with capacity constraint has a DSC structure and thus has a unique normalized equilibrium.

In our case we have

$$
g(x, r)=\left[\begin{array}{c}
\frac{r_{1} x_{-1}}{r_{2} x_{-2}} \\
X \\
\vdots \\
\frac{r_{N} x_{-N}}{X}
\end{array}\right]
$$


where $X=\sum_{i=1}^{N} x_{i}$ and $x_{-m}=\sum_{i=1, i \neq m}^{N} x_{i}$

$$
\begin{gathered}
G_{i, j}=\frac{\partial}{\partial x_{j}}\left(\frac{\partial}{\partial x_{i}} \frac{r_{i} x_{i}}{X}\right) \\
r_{i} \frac{\partial}{\partial x_{j}}\left(\frac{x_{-i}}{X^{2}}\right)= \begin{cases}r_{i} \frac{-2 x_{i}}{X^{3}} & \text { if } i=j \\
r_{i} \frac{x_{i}-x_{-i}}{X^{3}} & \text { if } i \neq j\end{cases}
\end{gathered}
$$

For $\left[G+G^{\prime}\right]$ consider

$$
G_{i, j}+G_{j, i}= \begin{cases}\frac{-4 r_{i} x_{-i}}{X^{3}} & \text { if } i=j \\ \frac{r_{i}\left(x_{i}-x_{-i}\right)+r_{j}\left(x_{j}-x_{-j}\right)}{X^{3}} & \text { if } i \neq j\end{cases}
$$

$\left[G+G^{\prime}\right]$ is negative definite if $A^{\prime}\left[G+G^{\prime}\right] A<0, \forall A, A \neq 0$ where $\mathrm{A}$ is the column vector

$$
\begin{gathered}
A=\left[\begin{array}{c}
a_{1} \\
\vdots \\
a_{N}
\end{array}\right] \\
A^{\prime}\left[G+G^{\prime}\right] A= \\
\sum_{i=1}^{N}\left[\sum_{j=1, j \neq i}^{N} a_{i} a_{j} \frac{r_{i}\left(x_{i}-x_{-i}\right)+r_{j}\left(x_{j}-x_{-j}\right)}{X^{3}}\right]-a_{i}^{2} \frac{4 r_{i} x_{-i}}{X^{3}}
\end{gathered}
$$

We choose $r_{i}=1$ for all $i$. Then (7) equals $-Z / X^{3}$ where $Z$ is given by

$$
\begin{aligned}
& \sum_{i=1}^{N} a_{i}^{2} 4 x_{-i}+\left[\sum_{j=1, j \neq i}^{N} a_{i} a_{j}\left(\left(x_{-i}-x_{i}\right)+\left(x_{-j}-x_{j}\right)\right)\right] \\
= & \sum_{i=1}^{N} a_{i}^{2} 4\left(X-x_{i}\right)+\left[\sum_{j>i}^{N} 4 a_{i} a_{j}\left(X-x_{i}-x_{j}\right)\right] \\
= & 4 \sum_{i=1}^{N} a_{i}^{2}\left(X-x_{i}\right)+\left[\sum_{j>i}^{N} a_{i} a_{j}\left(X-x_{i}-x_{j}\right)\right] \\
= & 4 \sum_{i=1}^{N}\left[a_{i}^{2} \sum_{j=1, j \neq i}^{N} x_{i}+\sum_{j>i}^{N} a_{i} a_{j} \sum_{k=1, k \neq j, k \neq i}^{N} x_{k}\right] \\
= & \sum_{i=1}^{N} 4 x_{i}\left[\sum_{j=1, j \neq i}^{N} a_{j}^{2}+a_{j} \sum_{k>j, k \neq i}^{N} a_{k}\right]
\end{aligned}
$$

Now (12) is positive for any positive value of $\mathrm{x}$ and hence $\left[G^{\prime}+G\right]$ matrix is negative definite.

\section{SEVERAL RESOURCES}

We consider next the following extension to the case of $K$ resources. Each player $m$ of the $M$ players has a budget $B(m)$ that he can invest by bidding $x_{k}^{m}$ of resource $k$. The following (orthogonal) constraint should hold:

$$
\sum_{k=1}^{K} x_{k}^{m} \leq B(m)
$$

The payoff for player $m$ is the sum of payoffs in all $K$ contests, i.e.

$$
P^{m}(x)=\sum_{k=1}^{K} P_{k}^{m}\left(x_{k}\right)
$$

where $x_{k}$ is the vector $x_{k}^{1}, \ldots, x_{k}^{M}$ and where

$$
P_{k}^{m}\left(x_{k}\right)=\frac{x_{k}^{m}}{\sum_{j=1}^{M} x_{k}^{j}} .
$$

and the cost of a contest $k$ to player $m$ is $\gamma(k) x_{k}^{m}$. Player $m$ 's utility is thus

$$
U^{m}(x)=\sum_{k=1}^{K}\left(\frac{x_{k}^{m}}{\sum_{j=1}^{M} x_{k}^{j}}-\gamma_{k} x_{k}^{m}\right)
$$

For the study of such games, see [17].

We next define capacity constraint on each of the $K$ resources. Let $V$ be the column vector with the $k$ th entry being a constant $V_{k}$. We then require for each $k$ that

$$
\sum_{m=1}^{N} x_{k}^{m} \leq V_{k}
$$

Note that when applying KKT conditions to the best response at equilibrium, we shall have $K$ Lagrange multiplyers. We wish to find a vector of $K$ Lagrange multiplyers that do not depend on the player nor on the policy of other players, such that the Nash equilibrium for the relaxed game will be an equilibrium for the original constrained game and in particular the constraints would be met and would satisfy the complentarity conditions. This is the vector version of a normalized equilibrium.

According to Theorem 4 of Rosen [15], we have to show that the set of utilities is diagonally strict concave in order to have existence and uniqueness of the normalized equilibrium. The latter follows from the fact that DSC holds for each resource separately and then apply the proof of Corollary 2 in [13].

\section{CONCLUSIONS AND FUTURE WORK}

We have shown that the utilities in the Tullock game are strict diagonal concave. This allows to conclude using Rosen's result that in absence of common correlated constraints, the Nash equilibrium exists and is unique, while in presence of such constraints, the normalized equilibrium exists in pure strategies and is unique. Note that while the statements on the Nash equilibrium have already been available in [1] which proposed an extension to the DSC property, that reference does provide tools to handle the normalized equilibrium.

Another advantage from the derivation of the DSC structure is that one can use dynamic distributed algorithms to converge to the normalized equilibrium and convergence is guaranteed under DSC, see [15]. 


\section{REFERENCES}

[1] Eitan Altman, Manjesh Kumar Hanawal, Rajesh Sundaresan. Generalising diagonal strict concavity property for uniqueness of Nash equilibrium. Indian Journal of Pure and Applied Mathematics, Indian National Science Academy, 2016, 47 (2), pp.213-228. ¡10.1007/s13226-016-0185-4i. ¡hal-01340963i

[2] Eitan Altman, Alexandre Reiffers-Masson, Daniel Sadoc Menasché, Mandar Datar, Swapnil Dhamal, Corinne Touati, "Mining competition in a multi-cryptocurrency ecosystem at the network edge: A congestion game approach", SOCCA 2018 - 1st Symposium on Cryptocurrency Analysis, Dec 2018, Toulouse, France. pp.1-4

[3] Eitan Altman and Eilon Solan, Constrained Games: the impact of the attitude to adversary's constraints, IEEE Transactions on Automatic Control, Oct. 2009, $54: 10$, pp 2435 - 2440. DOI: 10.1109/TAC.2009.2029302

[4] Eitan Altman and Adam Shwartz, (2000) Constrained Markov Games: Nash Equilibria. In: Filar J.A. Gaitsgory V., Mizukami K. (eds) Advances in Dynamic Games and Applications. Annals of the International Society of Dynamic Games, vol 5. Birkhauser, Boston, MA

[5] E. Altman, K. Avrachenkov, N. Bonneau, M. Debbah, R. El-Azouzi and D. Sadoc Menasche, "Constrained Cost-Coupled Stochastic Games with Independent State Processes", Operations Research Letters, Vol 36 pp. 160-164, 2008.

[6] G. Debreu, "A social equilibrium existence theorem", Proceedings of the National Academy of Sciences of the U.S.A, 38:886-893, 1952.

[7] R. El-Azouzi and E. Altman, "Constrained traffic equilibrium in routing", IEEE Transaction on Automatic Control, 48:1656-1660, 2003.

[8] E. Gomez-Ramırez, K. Najim and A.S. Poznyak, "Saddle-point cal- culation for constrained finite Markov chains". Journal of Economic Dynamics and Control, 27:1833-1853, 2003.

[9] M. T. Hsiao and A. A. Lazar. "Optimal decentralized flow control of Markovian queueing networks with multiple controllers", Performance Evaluation, 13:181-204, 1991.

[10] L. C. M. Kallenberg (1983), Linear Programming and Finite Markovian Control Problems, Mathematical Centre Tracts 148, Amsterdam.

[11] Krueger, Anne (1974). "The Political Economy of the Rent-Seeking Society". American Economic Review. 64 (3): 291-303. JSTOR 1808883

[12] T. Larsson and M. Patriksson. "Side constrained traffic equilibrium models - traffic management through link tolls". In P. Marcotte and S. Nguyen, editors, Equilibrium and Advanced Transportation Modelling, pages 125-151. Kluwer Academic Publishers, 1998.

[13] A. Orda, R. Rom and N. Shimkin, "Competitive routing in multi-user communication networks," IEEE/ACM Trans. on Networking 1(5), pp. 510-521, Oct. 1993.

[14] L. Pavel, "An extension of duality to a game-theoretic framework", Automatica, Volume 43 , Issue 2, pp. 226-237, 2007.

[15] J. Rosen, "Existence and uniqueness of equilibrium points for concave n-person games", Econometrica, 33:520-534, 1965.

[16] N. Shimkin, "Stochastic games with average cost constraints" in Annals of the International Society of Dynamic Games, MA, Boston:Birkhauser, vol. 1, 1994.

[17] Alexandre Reiffers-Masson, Yezekael Hayel and Eitan Altman, Game theory approach for modeling competition over visibility on social networks. 2014 6th International Conference on Communication Systems and Networks, COMSNETS 2014. 1-6. 10.1109/COMSNETS.2014.6734939.

[18] Martin Kaae Jensen, "Existence, Uniqueness and Comparative Statics in Contests", University of Leicester, Working Paper No. 15/16, July; 2015.

[19] Ferenc Szidarovszky and Koji Okuguchi, "On the Existence and Uniqueness of Pure Nash Equilibrium in Rent-Seeking Games", Games and Economic Behavior, 1997, vol. 18, issue 1, 135-140 